\title{
Environmental data and the Internet: openness and digital data management
}

\author{
Jørgen Tulstrup
}

In recent years the Geological Survey of Denmark and Greenland (GEUS) has, for a variety of reasons, worked intensively on implementing Internet technologies. The most important aim has been to provide public and private companies with access to many of the geological databases and maps at the Survey, thus substantially increasing the value of the geological data. In this way GEUS is implementing the intentions of the United Nations Aarhus-convention in respect of access to information, public participation in decision-making and access to justice in environmental matters (UNEC 1998). Another important objective has been to improve the procedures that deal with data input, registration and quality control of the large amounts of data that GEUS receives from regional authorities, private drilling companies and advisers, as well as that acquired by the activities of its own geological staff. This complies with the Danish Government's initiatives for making the public sector more efficient through digital data management.

\section{Openness}

During most of its lifetime the Survey has allocated substantial resources to the task of organising and securing the vast

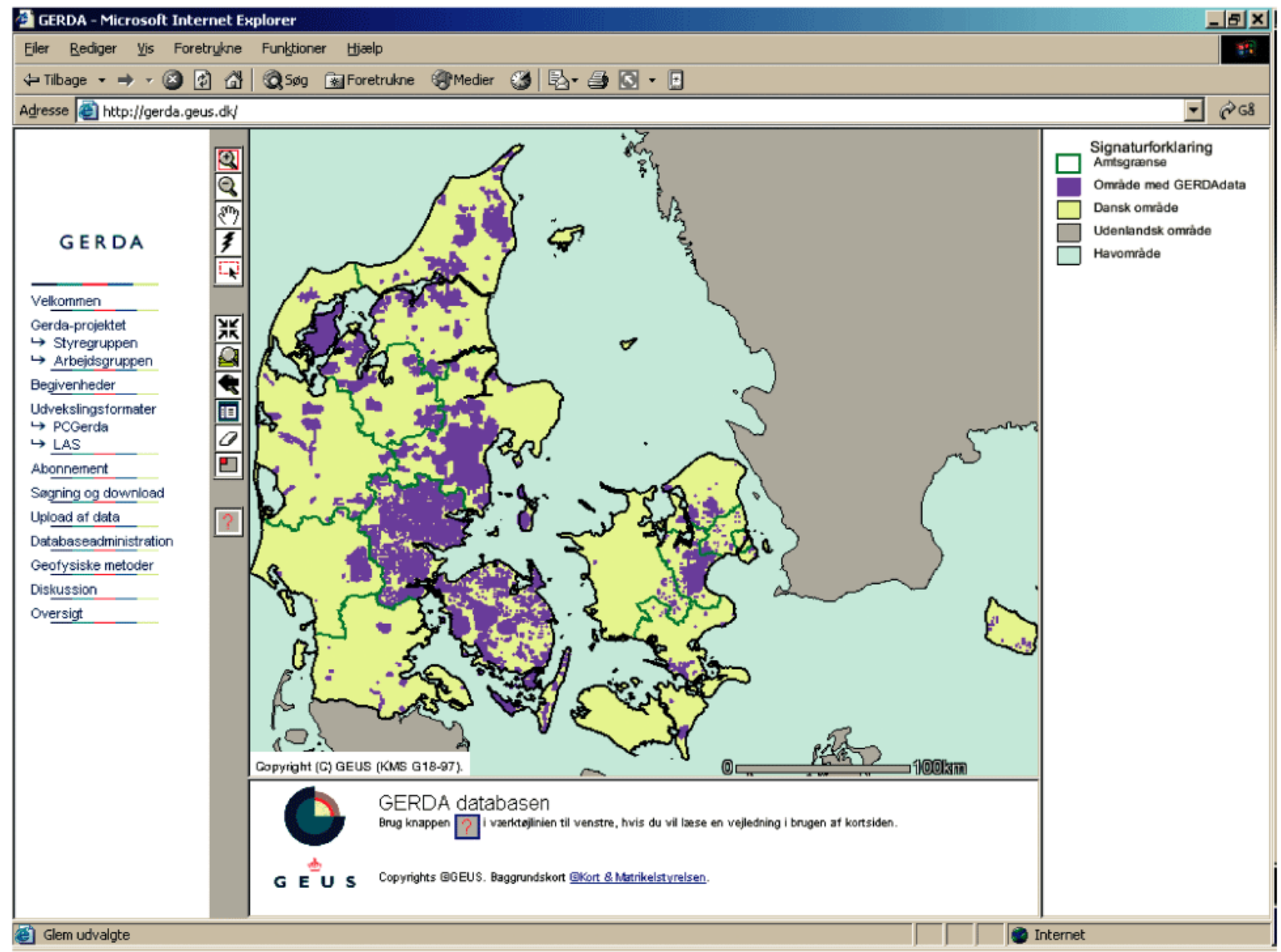

Fig. 1. Coverage of Danish shallow geophysical data from the Gerda database. 


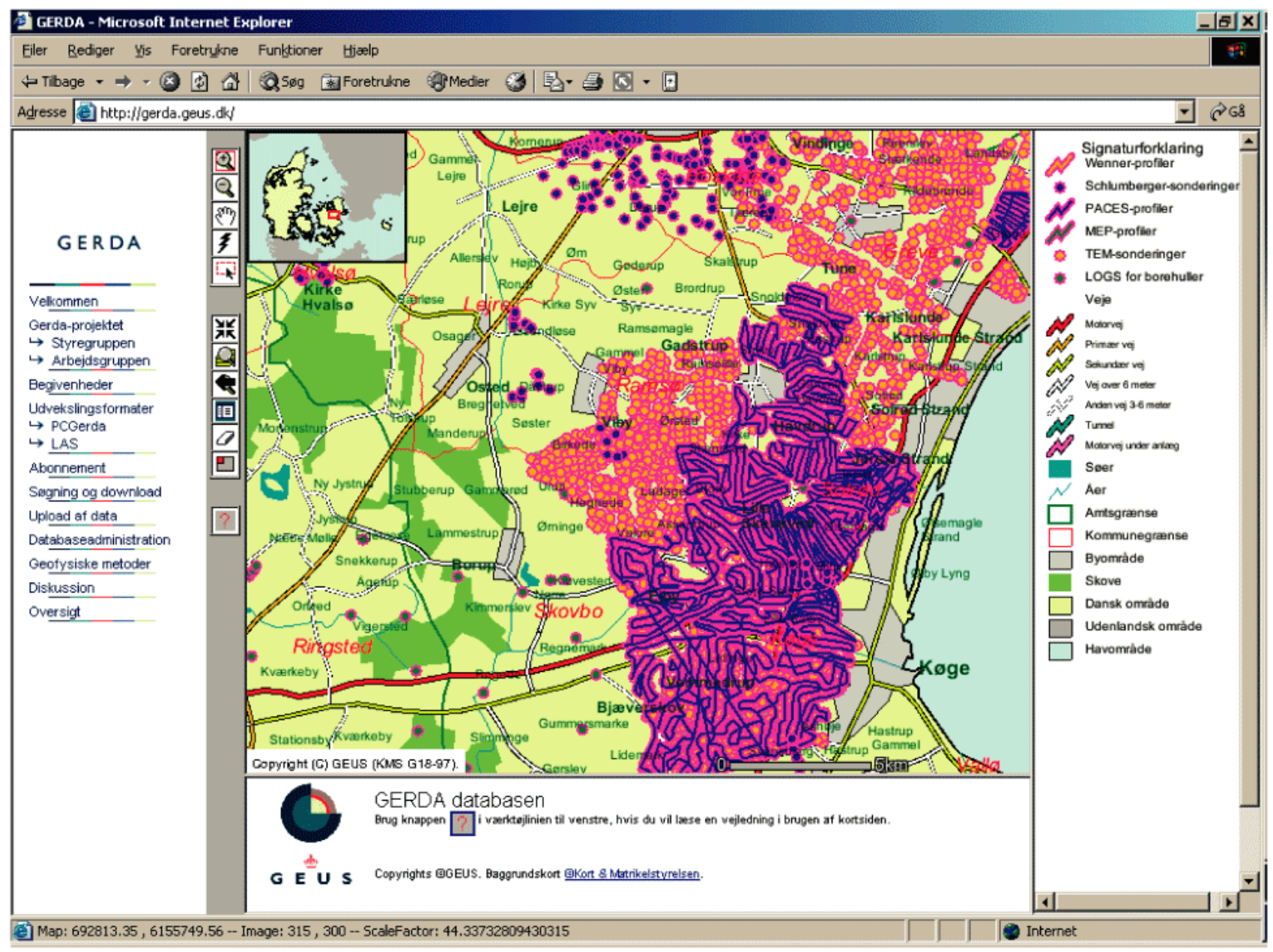

Fig. 2. Map available in a web-browser showing the position of Schlumberger soundings (purple/blue circles), pulled array continuous electrical soundings (purple/blue lines), transient electromagnetic soundings (red/orange circles) and boreholes with log-data (red/green circles). The GIS functionality is based on the ESRI product ArcIMS.

amount of geological data the Survey is responsible for as a national institution. In 1926 the Well Data Archive was established in response to a law passed by the Danish Parliament requiring that information from all new water supply wells should be reported to the Survey. Fifty years later the first version of a database for these data was built. Since then the database has undergone continuous development, including two major upgrades to new technology, and is now known as the Jupiter database. In the same period the quality of data has been improved considerably through close cooperation with the drilling companies who supply the data, and through quality control procedures at the Survey.

Jupiter is today a nationwide database designed for data relating to shallow boreholes, groundwater chemistry, water levels, water resources, etc. This database contains data for more than 230000 Danish boreholes and more than 5.7 million chemical analyses of groundwater and drinking water.
Another nationwide database called Gerda (Geophysical Relational Database) was established in 1999 and is designed for shallow geophysical data including geoelectrical profiles, geoelectrical soundings, electromagnetic soundings, borehole logs and geophysical models. This database contains geophysical data and interpretations for more than 600000 localities in Denmark.

Through the years great efforts have been made into making the data as useful and accessible as possible for internal as well as external users, continually updating the databases to keep pace with the increased quantity of data and continual improvements in technology. The widespread development of the Internet, and increased broad-band access, has made it possible to place data at the disposal of the general public and to make it available in an increasingly user-friendly and understandable way. 


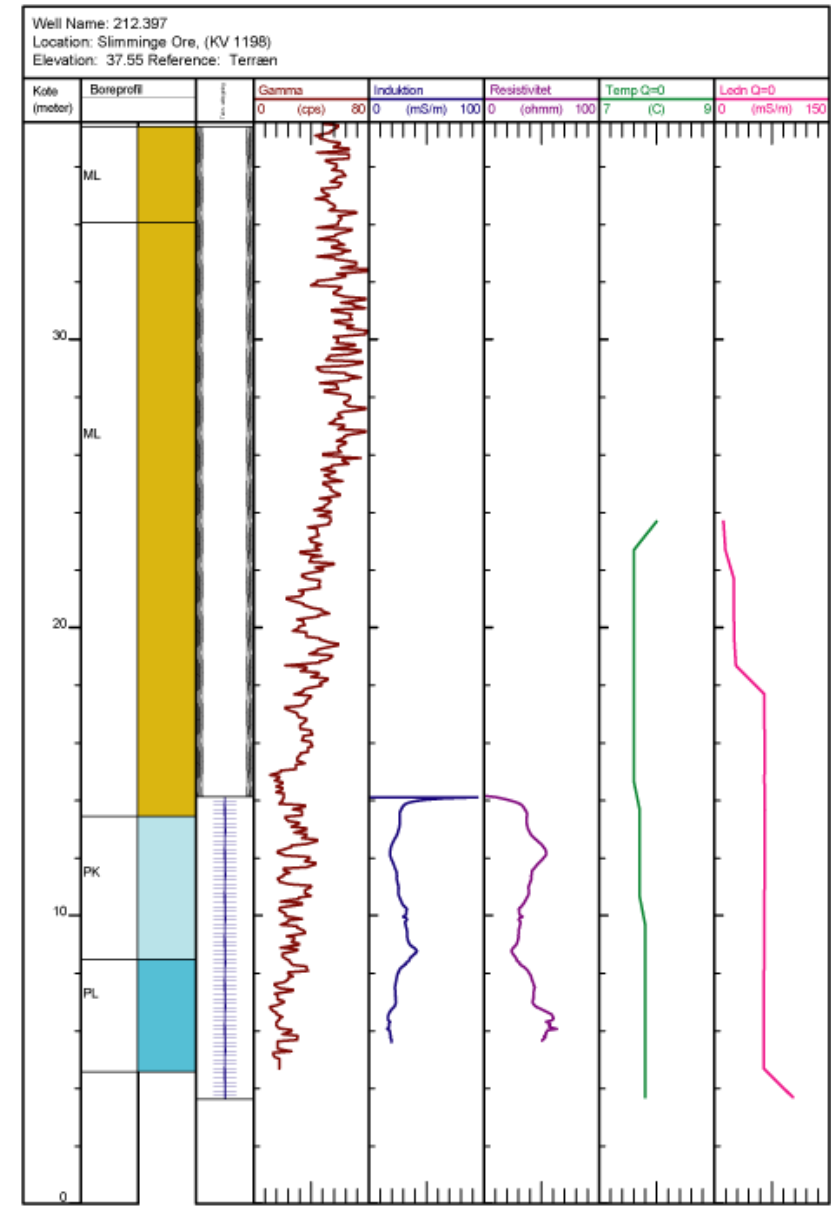

Fig. 3. Display of well-log data from the Gerda database. The graphics are available as a PDF file.

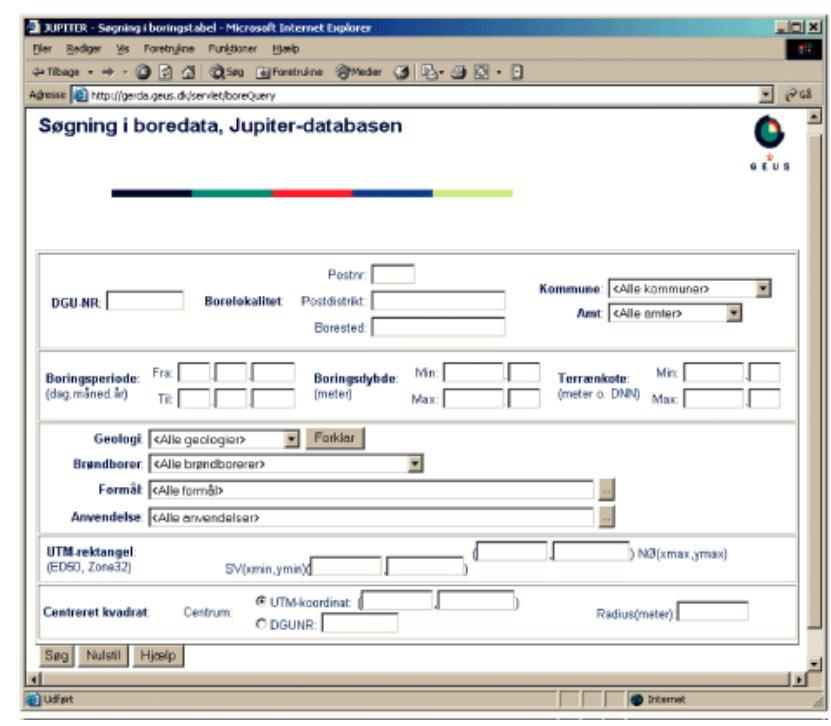

Fig. 4. Web-based search form for the Jupiter database. By entering search criteria the user can produce a list of boreholes. Each borehole on the resulting list can be examined in detail.
It is now possible to use a standard web browser to search for data utilising either Geographic Information System (GIS) facilities or a more traditional alphanumeric search. With the GIS interface (Fig. 1) it is possible to zoom, pan and choose an object on the map, e.g. a borehole or a sounding position (Fig. 2). By clicking the object the user can be guided to a website showing details of the object, for example a graphic illustration of a borehole log and a geological interpretation (Fig. 3).

Using the alphanumeric search (Fig. 4) the user will be presented with a list containing boreholes fulfilling different search criteria, e.g. name of drilling company, municipality, time of drilling, maximum hole depth, purpose of the borehole, or distance to a given point, as well as the possibility of searching on the unique borehole number. Furthermore, it is now possible to search for wells that penetrate certain geological horizons.

Another data type available on the Internet is a nationwide surface geological map (Fig. 5). This map of Denmark is based on field work that the Survey has carried out since 1890. The map shows the geology at a depth of one metre and was originally produced at the scale of 1:25 000 .

\section{Digital data management}

To reduce the steadily increasing cost related to registration and quality control of the geological data, a digital data management system has been introduced. Previously the necessary procedures included manually typing or scanning large amounts of data. However, since the data to be entered into the databases are often in a digital format when they are received, there is now the possibility of introducing complete or partly automated procedures in which computer programs handle quality control and loading of data into the databases. Closer manual inspection is only necessary when data quality is considered uncertain compared to existing data. The latest Internet technology also makes it possible for individual users to upload their own data.

The Gerda system was, from the outset, designed with the Internet in mind. The data suppliers, such as geophysical companies, are able to directly upload data files. The data are quality controlled and then loaded into the database. An email message to the supplier supplies information on the progress of the procedure. After this is complete, an updated database is extracted from the central Gerda database and made available to users of the data, e.g. geologists in the Danish counties.

Uploaded data files in Gerda contain information about projects, recording instruments, etc. This information is accessible to the data suppliers, enabling them to create and 


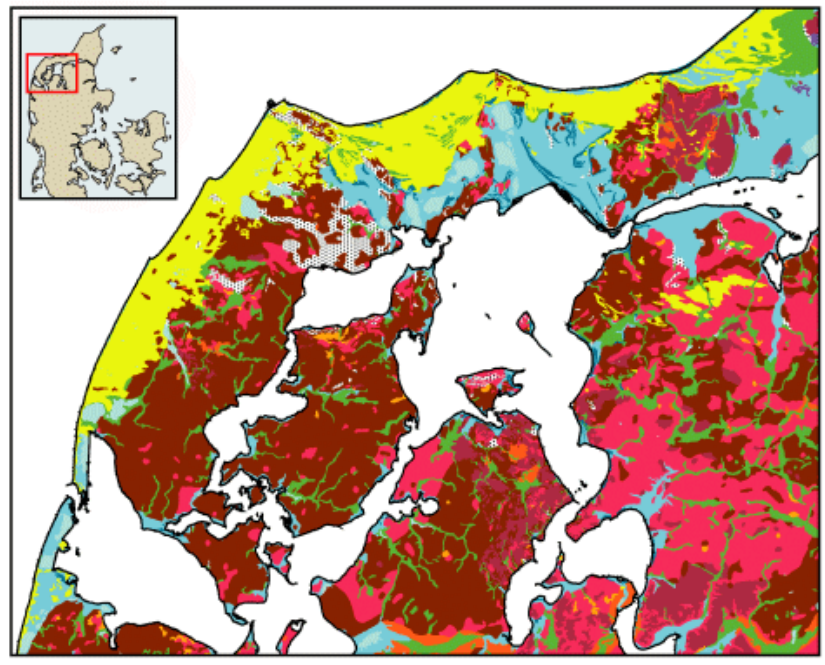

Fig. 5. Example of surface geological map of Denmark available at the GEUS website.

maintain data directly on the website. The procedure ensures a very flexible and non-bureaucratic work routine.

In 1999 GEUS merged four older databases into a single database (Jupiter) to obtain a much higher level of automated treatment of the collected data. This has made it possible for GEUS to receive digital data reports of groundwater and drinking water quality, water resources and water level observations from the districts.

The data are received in the so-called 'STANDAT' format (generally used for exchange of environmental data in Denmark) and is converted into the XML-format (Extensible Markup Language). When completed, a comprehensive quality control is undertaken. The supplier automatically receives a load report by e-mail. The report is a detailed description of syntax errors, data errors, data exceeding existing limits, and information on whether substance concentrates have been registered to be diverging substantially from previous measurements.

In the near future it will also be possible to accept basic borehole data digitally. GEUS has developed a program, PC Jupiter, which will be used by the drilling companies for plan- ning new water supply boreholes, as well as for registration of well localities, the technical data of the borehole, the observed geological stratification and test pumping. The program stores the data in a database administered locally by the drilling company. The data are also forwarded to GEUS where the data are validated and finally loaded into the central Jupiter database.

\section{Web-services}

The Internet solutions described above are designed mainly to make data accessible on the GEUS website using traditional HTML-technology.

To further increase the accessibility of the geological data, a new group of technologies known as web-services are under implementation. A web-service is a function available on a web-server, which on the request of a user or another webserver delivers data in XML-format, or maps as images (Web Map Services). This technology will enable other authorities and companies who set up web-servers to make use of geological data without having to be concerned about keeping local copies of data complete and up-to-date. The data are retrieved on-line from GEUS' systems.

An example of this is a solution GEUS is providing for an Internet portal that the Danish counties are implementing. The portal provides access to a wide range of environmental data including borehole, groundwater and drinking water data. The portal will make it possible for users to view data on a map and to display graphs of time series and download data. The data will be retrieved live from the Jupiter database through a web-service interface. Data input to Jupiter from the regional authorities will also take place through web-services.

\section{Reference}

UNEC 1998: The Aarhus Convention. Resolution on access to information, public participation in decision-making and access to justice in environmental matters, Fourth Ministerial Conference 'Environment for Europe', Aarhus, Denmark, 23-25 June 1998. United Nations Economic Commission for Europe, 4 pp. 